\title{
ASSOCIAÇÃO ENTRE A IDADE À PRIMEIRA COBERTURA DA MARRÃ E SEU DESEMPENHO REPRODUTIVO
}

Tatiane Marquini Ribeiro ${ }^{1 *}$; Adriel Silva Tavares Azevedo²; Jessica Silva Soares ${ }^{3}$; Ednaldo Carvalho Guimarães ${ }^{4}$; Robson Carlos Antunes ${ }^{5}$.

${ }^{1}$ Graduanda em Medicina Veterinária na Universidade Federal de Uberlândia, Uberlândia-MG, Brasil, tatiane_m_r@hotmail.com;

${ }^{2}$ Graduando em Medicina Veterinária na Universidade Federal de Uberlândia, Uberlândia -MG, Brasil;

${ }^{3}$ Mestranda em Ciências Veterinárias na Universidade Federal de Uberlândia

(FAMEV/UFU), Uberlândia-MG, Brasil;

${ }^{4}$ Professor Doutor na Universidade Federal de Uberlândia (FAMAT/UFU), Uberlândia-MG, Brasil;

${ }^{5}$ Professor Doutor na Universidade Federal de Uberlândia (FAMEV/UFU), Uberlândia-MG, Brasil.

Recebido em: 06/04/2019 - Aprovado em: 10/06/2019 - Publicado em: 30/06/2019 DOI: 10.18677/EnciBio_2019A22

A suinocultura industrial vem crescendo atualmente, todavia há uma linha tênue no quesito margem lucrativa. O preparo da leitoa de reposição é de extrema importância para a obtenção de bons resultados, pois tais matrizes serão responsáveis pelo fornecimento das próximas leitegadas. A idade da primeira cobertura de marrãs pode estar diretamente relacionada com a produtividade destas. No entanto, não há um consenso preciso acerca da idade ideal para a primeira cobertura. Objetivou-se com este estudo relacionar a idade à primeira cobertura de marrãs com a produtividade destas nos partos posteriores. Foram utilizados os dados reprodutivos dos quatro primeiros partos de 111 fêmeas Large White. Levou-se em consideração informações sobre a quantidade de nascidos vivos, mortos e mumificados em cada parto/gestação; a idade a primeira cobertura; o número de leitões desmamados; e o peso médio dos leitões ao nascer e ao desmame. As variáveis peso médio ao nascer no parto 1 e número total de nascidos vivos nos quatros partos apresentaram correlação significativa. Fêmeas cobertas abaixo de 215 dias apresentaram menor mumificação fetal no primeiro parto e nos partos subsequentes. No entanto, fêmeas cobertas entre 235 e 279 dias apresentam peso ao nascer maior no primeiro parto.

PALAVRAS-CHAVE: Produtividade, Reprodução, Suínos. 


\title{
ASSOCIATION BETWEEN AGE OF GILTS AT FIRST MATING AND THEIR REPRODUCTIVE PERFORMANCE
}

\begin{abstract}
The Industrial swine farming is currently growing, but there is a thin line when it comes to the lucrative margin. The preparation of the replacement gilt is extremely important for the obtention of good results, since these matrices will be responsible for supplying the next litter. The age of the gilt when it's covered for the first time may be directly related to their productivity. However, there is no precise consensus about the ideal age for the first coverage. The goal of this study was to relate the age of the first coverage of gilts with the productivity of these in later deliveries. The reproductive data of the first four deliveries of 111 Large White females were used. The data that was taken into consideration was about the number of piglets that were born alive, dead and mummified in each birth/gestation; the age of the first coverage; the number of weaned piglets; and the average weight of piglets at birth and weaning. The variables average birth weight on the first calving and total number of live births in the four deliveries showed a significant correlation. Females covered below 215 days presented lower fetal mummification in the first calving and subsequent deliveries. However, females covered between 235 and 279 days present higher birth weight in the first calving.
\end{abstract}

KEYWORDS: Productivity, Reproduction, Swine.

\section{INTRODUÇÃO}

A carne suína é a segunda proteína animal mais consumida no mundo, ficando atrás apenas dos pescados, e o consumo de carne suína vem crescendo significativamente no Brasil e no mundo (GUIMARÃES et al., 2017).

É claramente reconhecido que a produtividade do rebanho reprodutivo é um dos principais fatores que determinam a lucratividade da indústria suinícola (KANEKO; KOKETSU, 2012). A otimização do gerenciamento da marrã é um ponto crítico para melhorar a eficiência do rebanho reprodutivo, sendo assim, as práticas tradicionais de manejos devem ser reavaliadas com atenção aos diferentes genótipos modernos (BORTOLOZZO et al., 2009), afinal apesar do cenário da suinocultura indicar um modelo de negócio que sofre constantes mudanças, vem apresentando uma margem lucrativa cada vez menor (BORTOLOZZO et al. 2015), portanto é necessário que médicos veterinários saibam os fatores que afetam o desempenho reprodutivo da porca, a fim de maximizar o potencial de cada animal e otimizar a produtividade do plantel (KOKETSU et al., 2017).

Uma das condições de alta eficiência na produção de suínos é a reprodução bem-sucedida (BABICZ et al., 2019). A variação na fertilidade das marrãs está associada ao aumento da reposição e à redução da longevidade (KNOX et al., 2016). Há um aumento da demanda econômica por marrãs com ótimo potencial reprodutivo e longevidade, portanto, o futuro do rebanho de matrizes e o sucesso das granjas de suínos é amplamente baseado em marrãs de reposição. Qualquer tentativa de aumentar seu potencial reprodutivo, melhorar sua saúde e prolongar sua longevidade, é bem vinda e gera um grande impacto econômico benéfico na indústria (ROY et al., 2016). Calcula-se que 40 a 50\% das matrizes sejam abatidas anualmente, sendo cerca de metade desses abates associadas à primeira paridade da porca ou à substituição destas (ENGBLOM et al., 2008).

Por conseguinte, estratégias de reposição de leitoas afetam diretamente os índices da granja, devido ao alto impacto que representam nos desempenhos 
reprodutivo e produtivo de um sistema de produção de suínos, portanto, para se alcançar um número ideal de leitões desmamados por ano por fêmea, é necessário que esta tenha uma distribuição de ordem de partos ideal, sendo assim, a taxa anual de entrada de leitoas de reposição no plantel deve ser correta e nunca negligenciada (ANTUNES, 2018). Além disso, o número de dias a partir do ingresso da fêmea na granja até o momento da cobertura é o período que mais contribui para o aumento de um importante indicador reprodutivo negativo: os dias não produtivos (DNP), que ocorrem quando a matriz não está nem gestando, nem lactando (ANTUNES, 2018).

A duração da vida produtiva da porca possui correlação genética moderada com a idade ao primeiro parto (SAITO et al., 2011), ou seja, pode-se concluir que a idade ao primeiro estro das marrãs de substituição influencia significativamente seu desempenho reprodutivo subsequente (ROONGSITTHICHAI et al., 2013), sendo assim, o reconhecimento do momento apropriado para inseminação artificial ou acasalamento natural de porcas é um dos elementos-chave da reprodução efetiva de suínos (BABICZ et al., 2019). No entanto, as recomendações à primeira cobertura variam de acordo com as características genéticas, sendo observado principalmente a idade, o peso, o número de cios apresentados antes da primeira cobertura e a espessura do toucinho (KUMMER et al., 2005), não sendo definida uma idade ótima para a primeira cobertura, que coincida com as recomendações de todas as empresas de melhoramento genético de suínos. Além disso, as marrãs mais novas no início do estro de cobertura apresentam maior taxa de partos e vida reprodutiva mais longa do que aquelas com puberdade tardia (SAITO et al., 2011). Portanto, as marrãs de substituição devem ter alta taxa de crescimento e entrar em contato com o cachaço mais cedo para atingir a puberdade mais rapidamente e possuir o desempenho reprodutivo subsequente desejável (ROONGSITTHICHAI et al., 2014).

Enfim, práticas de gestão que aumentam a proporção de leitoas com menor idade a primeira cobertura dentro de um rebanho são importantes dentro da produção de suínos (SAITO et al., 2011), afinal a substituição de leitoas por estro no tempo previsto permite um agendamento e uso mais eficiente das instalações de gestação e parto da granja e proporciona maior oportunidade para a introdução de fêmeas em grupos de porcas no momento em que as mesmas são manejadas em baias coletivas após o desmame (CASSAR, 2009).

Com o presente estudo objetivou-se correlacionar a idade à primeira cobertura das marrãs com a produtividade destas ao longo de suas vidas reprodutivas, buscando estabelecer uma idade ótima para a primeira cobertura, na qual os índices reprodutivos nos primeiros quatro partos atinjam os maiores níveis possíveis, pois, segundo Pinilla e Lecnieski (2010), para ocorrer amortização do custo de produção de uma fêmea, são necessários três partos, já para obter retorno financeiro sobre o investimento, são necessários no mínimo quatro partos, além disso, estudos afirmam que o abate da matriz após a quarta paridade gera porcos desmamados mais vendáveis no sistema de produção (GRUHOT et al., 2017).

\section{MATERIAL E MÉTODOS}

\section{Local e animais}

A coleta de dados foi realizada em uma granja multiplicadora no município de Passos, no estado de Minas Gerais - Brasil, latitude 204' 08” S, longitude 46 $36^{\prime}$ 36" O. Foram utilizados dados reprodutivos de 111 fêmeas suínas da raça Large White até o $4^{\circ}$ parto. Levou-se em consideração informações sobre a quantidade de 
nascidos vivos, mortos e mumificados em cada parto/gestação; a idade da primeira cobertura; o número de leitões desmamados; e o peso médio dos leitões ao nascer e ao desmame.

\section{Análise estatística}

A análise estatística dos dados foi realizada através do programa IBP SSPSS Statistics ${ }^{\circledR}$ Version 22, utilizando-se também ferramentas do programa Microsoft Excel. A significância das correlações foi calculada com o pacote estatístico computacional SISVAR (FERREIRA, 2011) utilizando procedimentos de Banzato e Kronka (2006). Para as características que apresentaram normalidade para ShapiroWilk (significância de $p<0,05$ ), foi aplicada a Correlação de Pearson, e para as que não apresentaram, foi aplicada a Correlação de Spearman.

Posteriormente, os dados das 111 marrãs foram distribuídos em três grupos, de acordo com a faixa etária. Os grupos foram os seguintes: G1 de 215 dias a 233 dias; G2 de 235 a 279 dias; e G3 de 281 a 314 dias. A escolha dos grupos baseouse no modelo de G1 abaixo da média -1 desvio padrão (14\% da amostra); G3 acima da média +1 desvio padrão (16\% da amostra); e G2 o intervalo entre G1 e G3 (70\% da amostra). Foi realizada análise descritiva dos grupos etários, obtendo-se os valores de média, mediana, mínimo e máximo, e desvio padrão destes. Realizou-se também uma análise de variância e teste de médias. Para os dados que preencheram os pressupostos de normalidade, antes ou após a transformação de dados, aplicou-se o teste de Tukey, caso contrário, o teste de Kruskal-Wallis.

\section{RESULTADOS E DISCUSSÃO}

As variáveis que tiveram correlação significativa com IPC (idade à primeira cobertura) foram PMNP1 (peso médio ao nascer no parto 1) $(p=0,015)$ e NVPt (total de nascidos vivos nos quatro partos) $(p=0,020)$ (Tabela 1$)$, resultados que diferiram de Roongsitthichai et al. (2013), no qual não registram diferença significativa no número de leitões nascidos vivos e nascidos totais. Pode-se inferir que, apesar das correlações obtidas neste estudo serem estatisticamente baixas, para $p<0,05$, o fato de NVPt ser significativo para Correlação de Spearman (Tabela 2) infere que quanto mais velha a fêmea suína for inseminada menor o peso médio dos leitões ao nascer no primeiro parto e menor o número de nascidos vivos ao longo dos quatro primeiros partos desta fêmea, resultado que contradiz o descrito em Vidović et al. (2011) e Szulc et al. (2015), estudos nos quais fêmeas mais velhas obtiveram melhores resultados reprodutivos, como por exemplo uma maior quantidade de leitões nascidos vivos.

Tal fato pode significar perda econômica ao se esperar mais tempo para realizar-se a primeira cobertura das marrãs, pois a eficiência da produção de suínos é determinada, entre outros fatores, pelo número de leitões obtidos por porca por ano (GONZALEZ-ANOVER; GONZALEZ-BULNES, 2017). No entanto, a idade a primeira cobertura é sugerida como um indicador de desempenho reprodutivo ao longo da vida, tem sido demostrado que marrãs acasaladas mais jovens são abatidas mais tarde quando comparado à marrãs cobertas com idade mais avançada (MORRISON, 2016). A menor idade ao primeiro parto tem o potencial de aumentar a retenção de matrizes no rebanho e diminuir o número de fêmeas de reposição, sendo este aspecto econômico importante na cadeia suinícola (MORRISON, 2016; SEE et al., 2018). 
TABELA 1- Correlação de Pearson: IPC x variável, normais para Shapiro-Wilk $(p<0,05)$.

\begin{tabular}{|c|c|c|c|c|c|}
\hline Variável & Pearson & p-valor & Variável & Pearson & p-valor \\
\hline NVP1 & $-0,117$ & 0,222 & DESP3 & 0,005 & 0,959 \\
\hline NMP1 & 0,103 & 0,282 & PMNP3 & $-0,044$ & 0,644 \\
\hline MMP1 & 0,094 & 0,282 & NVP4 & $-0,080$ & 0,404 \\
\hline DESP1 & $-0,178$ & 0,061 & NMP4 & 0,033 & 0,731 \\
\hline PMNP1 & 0,231 & 0,015 & MMP4 & 0,152 & 0,113 \\
\hline PMDP1 & 0,139 & 0,146 & DESP4 & 0,135 & 0,157 \\
\hline GPMP1 & 0,102 & 0,287 & PMNP4 & 0,093 & 0,333 \\
\hline NVP2 & $-0,058$ & 0,544 & NMPt & 0,128 & 0,181 \\
\hline NMP2 & 0,135 & 0,158 & MMPt & 0,068 & 0,480 \\
\hline MMP2 & $-0,075$ & 0,433 & DESPt & $-0,027$ & 0,776 \\
\hline DESP2 & $-0,029$ & 0,766 & P1Tn & $-0,027$ & 0,781 \\
\hline PMNP2 & 0,106 & 0,268 & P2Tn & $-0,031$ & 0,750 \\
\hline NVP3 & $-0,144$ & 0,130 & P3Tn & $-0,117$ & 0,223 \\
\hline NMP3 & 0,003 & 0,974 & P4Tn & 0,018 & 0,851 \\
\hline MMP3 & 0,029 & 0,763 & & & \\
\hline
\end{tabular}

Sendo: IPC - idade na primeira cobertura; NVP1 - número de nascidos vivos no parto 1; NMP1 número de nascidos mortos no parto 1; MMP1 - número de mumificados no parto 1; DESP1 - número de desmamados no parto 1; PMNP1 - peso médio ao nascer no parto 1; PMDP1 - peso médio ao desmame no parto 1; GPMP1 - ganho de peso médio do nascer ao desmame no parto 1; NVP2 número de nascidos vivos no parto 2; NMP2 - número de nascidos mortos no parto 2; MMP2 número de mumificados no parto 2; DESP2 - número de desmamados no parto 2; PMNP2 - peso médio ao nascer no parto 2; NVP3 - número de nascidos vivos no parto 3; NMP3 - número de nascidos mortos no parto 3; MMP3 - número de mumificados no parto 3; DESP3 - número de desmamados no parto 3; PMNP3 - peso médio ao nascer no parto 3; NVP4 - número de nascidos vivos no parto 4; NMP4 - número de nascidos mortos no parto 4; MMP4 - número de mumificados no parto 4; DESP4 - número de desmamados no parto 4; PMNP4 - peso médio ao nascer no parto 4; NMPt - número de nascidos mortos total dos quatro partos; MMPt - número de mumificados total dos quatro partos; DESPt - número de desmamados total dos quatro partos; P1Tn - total de nascidos no parto 1, somando vivos, mortos e mumificados; P2Tn - total de nascidos no parto 2, somando vivos, mortos e mumificados; P3Tn - total de nascidos no parto 3, somando vivos, mortos e mumificados; P4Tn - total de nascidos no parto 4 , somando vivos, mortos e mumificados. 
TABELA 2- Correlação de Spearman: IPC X variável $(p<0,05)$.

\begin{tabular}{l|r|r}
\hline \multicolumn{1}{c|}{ VARIÁVEL } & Spearman & \multicolumn{2}{l}{-valor } \\
\hline PMDP2 & $-0,033$ & 0,732 \\
\hline GPMP2 & $-0,039$ & 0,684 \\
\hline PMDP3 & $-0,027$ & 0,775 \\
\hline GPMP3 & $-0,024$ & 0,800 \\
\hline PMDP4 & $-0,171$ & 0,072 \\
\hline GPMP4 & $-0,181$ & 0,057 \\
\hline NVPt & $-0,220$ & 0,020 \\
\hline PMNPt & 0,138 & 0,149 \\
\hline PMDPt & $-0,036$ & 0,707 \\
\hline MGPMPt & $-0,067$ & 0,487 \\
\hline P1+P2Tn & $-0,078$ & 0,414 \\
\hline P1+P2+P3Tn & $-0,112$ & 0,241 \\
\hline P1+P2+P3+P4Tn & $-0,095$ & 0,323 \\
\hline
\end{tabular}

Sendo: IPC - idade na primeira cobertura; PMDP2 - peso médio ao desmame no parto 2; GPMP2 - ganho de peso médio do nascer ao desmame no parto 2; PMDP3 - peso médio ao desmame no parto 3; GPMP3 - ganho de peso médio do nascer ao desmame no parto 3; PMDP4 - peso médio ao desmame no parto 4; GPMP4 ganho de peso médio do nascer ao desmame no parto 4; NVPt - número de nascidos vivos total dos quatro partos; PMNPt - peso médio ao nascer total dos quatro partos; PMDPt - peso médio ao desmame total dos quatro partos; MGPMPt média de ganho de peso médio total dos quatro partos; P1+P2Tn - soma do total de nascidos nos partos 1 e 2, somando vivos, mortos e mumificados; P1+P2+P3Tn soma do total de nascidos nos partos 1,2 e 3 , somando vivos, mortos e mumificados; P1+P2+P3+P4Tn - soma do total de nascidos nos partos 1, 2, 3 e 4, somando vivos, mortos e mumificados.

Analisando os dados em grupos observa-se que no grupo G1, coberto mais jovem (215-233 dias) não houve nenhum nascido mumificado no quarto parto, em nenhuma das fêmeas analisadas no presente estudo (Tabela 3), o que demonstra 
uma tendência na diminuição da quantidade de mumificados ao longo da vida reprodutiva da porca. Tal fato se consolida ao observar-se o MMPt (mumificados totais nos quatro partos), pois esta variável segue uma curva de aumento do primeiro grupo ao terceiro, sendo suas médias indo de 0,8 no $\mathrm{G} 1$, passando por 1,4 no G2 e terminando em 1,5 no G3 (Tabela 3), mostrando que quando cobertas mais velhas as fêmeas tendem a apresentar uma maior quantidade de leitões mumificados ao longo de suas vidas reprodutivas. Tal fato corrobora com a questão de que saltar cio de marrãs mais velhas pode levar a perda reprodutiva destas porcas por anestro (MOURA, 2016), justificando assim uma primeira cobertura em uma faixa etária mais jovem. Além do mais, de acordo com Almeida et al., (2017), há diferenças significativas no desenvolvimento do folículo ovariano de fêmeas cobertas mais velhas ou mais jovens, já que o peso dos leitões ao nascer altera a dinâmica do folículo em pré-púberes.

Todavia, um fator que favorece uma alta IPC (idade na primeira cobertura) é o peso médio ao nascer no parto um (PMNP1), que se mostrou maior no grupo G3 (Tabela 3) que continha fêmeas cobertas mais velhas, indicando que os leitões nascem mais pesados no primeiro parto destas, fato que pode ser justificado uma vez que esta já não necessita de tantos nutrientes para seu próprio crescimento, disponibilizando uma quantidade maior aos fetos. Além disso, o útero e o oviduto em porcas mais velhas está mais desenvolvido, proporcionando mais espaço para o crescimento e desenvolvimento dos leitões (KAPELAŃSKI et al., 2013) e quanto maior a capacidade uterina, maior o tamanho da leitegada (FREKING et al., 2016), somando-se o fato de que porcas mais velhas são capazes de manter e criar um maior número de leitões durante o período de lactação (GRUHOT et al., 2017); levando-se a refletir quanto a possibilidade de uma primeira cobertura mais velha ser mais viável ao visar-se um retorno financeiro mais significativo já na primeira ordem de parto.

TABELA 3- Análise descritiva dos grupos etários G1 G2

G3

\begin{tabular}{|c|c|c|c|c|c|c|c|c|c|}
\hline $\begin{array}{c}\text { Variáv } \\
\text { el }\end{array}$ & $\bar{x}$ & DP & Med & $\bar{x}$ & DP & Med & $\bar{x}$ & DP & Med \\
\hline \multirow{2}{*}{ IPC* } & 226,3 & \multirow{2}{*}{5,53} & \multirow{2}{*}{$\begin{array}{c}229,0 \\
\quad c\end{array}$} & 253,4 & 13,5 & \multirow{2}{*}{$\begin{array}{c}249,0 \\
b\end{array}$} & 295,9 & 11,0 & \multirow{2}{*}{$\begin{array}{c}294,5 \\
a\end{array}$} \\
\hline & 3 & & & 5 & 7 & & 4 & 0 & \\
\hline $\begin{array}{c}\text { PMNP1 } \\
\star\end{array}$ & 1,22 & 0,18 & $1,20^{b}$ & 1,24 & 0,18 & $1,20^{b}$ & 1,39 & 0,21 & $1,30^{a}$ \\
\hline $\mathrm{MMPt}^{*}$ & 0,80 & 0,86 & $1,00^{a}$ & 1,40 & 1,76 & $1,00^{a}$ & 1,50 & 1,43 & $1,00^{a}$ \\
\hline \multicolumn{10}{|c|}{$\begin{array}{l}\text { *Grupos seguidos de mesma letra não diferem entre si pelo teste de Kruskal-Wallis } \\
\text { com } 5 \% \text { de significância. Sendo: } \bar{x} \text { : média; DP: desvio padrão; Med: mediana; IPC - } \\
\text { idade na primeira cobertura; PMNP1 - peso médio ao nascer no parto 1; MMPt - } \\
\text { número de mumificados total dos quatro partos. Obs: Foram representados apenas } \\
\text { variáveis que apresentaram resultados significativos neste estudo. }\end{array}$} \\
\hline
\end{tabular}




\section{CONCLUSÃO}

Conclui-se que o IPC (Idade a primeira cobertura) é uma variável que apresenta influência direta no desempenho reprodutivo da marrã e em seus partos subsequentes. Fêmeas cobertas entre 215 e 233 dias de idade apresentam menor mumificação fetal no primeiro parto e nos partos subsequentes e um total de nascidos vivos maior ao longo de sua vida reprodutiva. No entanto, fêmeas cobertas entre 235 e 279 dias apresentam peso ao nascer maior no primeiro parto. Sendo assim, uma primeira cobertura em idade mais jovem (215-233 dias) mostra-se mais eficaz e uma premissa de uma vida reprodutiva mais vantajosa. Todavia, deve-se pesquisar a fundo sobre investimentos em leitoas de reposição e povoamento, contrabalanceando gastos em se manter a mesma no período de quarentena, e perdas e ganhos econômicos com o adiantamento ou atraso da primeira cobertura.

\section{REFERÊNCIAS}

ALMEIDA, F.R.C.L.; ALVARENGA-DIAS, A.L.N.; MOREIRA, L.P.; FIÚZA, A.T.L.; CHIARINI-GARCIA, H.. Ovarian follicle development and genital tract characteristics in diferente birthweight gilts at 150 days of age, Reproduction in Domestic Animals, v. 00, p. 1-7 - 2017. Disponível em: <https://doi.org/10.1111/rda.12976>. DOI: $10.1111 /$ rda12976.

ANTUNES, R.C.. O ensino da produção industrial de suínos - Uma visão crítica. $1^{1 \underline{a}}$ ed./ Uberlândia - MG: Edibrás - 2018.

BABICZ, M.; PASTWA, M.; SKRZYPCZAK, E.; ŁUSZCZEWSKA-SIERAKOWSKA, I.; WAWRZYNIAK, A.. Sexual activity of different gilt genotype groups of the Pulawska breed during oestrus. Medycyna Weterynaryjna, v. 1, n. 75, p. 50-53 - 2019. Disponível

em: <http://www.medycynawet.edu.pl/images/stories/pdf/pdf2019/012019/2019016165.pd f>. DOI: dx.doi.org/10.21521/mw.6165.

BANZATTO, D.A.; KRONKA, S.N.; Experimentação Agrícola. 4ª ed. Jaboticabal: Funep. p. 237 - 2006.

BORTOLOZZO, F.P.; BERNARDI, M.L.; KUMMER, R.; WENTZ, I.. Growth, body state and breeding performance in gilts and primiparous sows. Society for Reproduction and Fertility Supplement. v. 66, p. 281-91 - 2009. Disponível em: $<$ https://europepmc.org/abstract/med/19848294>. PMID: 19848294.

BORTOLOZZO, F.P.; FACCIN, J.E.G.; LASKOSKI, F.; MELLAGI, A.P.G.; BERNARDI, M.L.; WENTZ, I.. Desafios e potencialidades para o manejo reprodutivo da fêmea suína, Revista Brasileira Reprodução Animal, v.39, n.1, p. 97-103 2015. Disponível em: <http://cbra.org.br/pages/publicacoes/rbra/v39n1/pag97103\%20(RB540).pdf>.

CASSAR, G.. Hormonal Control of Pig Reproduction. London Swine Conference Tools of the Trade, v. 1-2, p. 137-139 - 2009. Disponível em: $<$ http://citeseerx.ist.psu.edu/viewdoc/summary?doi=10.1.1.488.9595>. $\quad$ DOI: 10.1.1.488.9595. 
ENGBLOM, L.; LUNDEHEIM, N.; STRANDBERG, E.; MDEL, P.S.; DALIN, A.M.; ANDERSSON, K.. Factors affecting length of productive life in Swedish commercial sows. Journal of Animal Science, v.86, p. 432-441 - 2008. Disponível em: <https://doi.org/10.2527/jas.2007-0310>. DOI: 10.2527 / jas.2007-0310.

FERREIRA, D.F.. Sisvar: a computer statistical analysis system. Ciência e Agrotecnologia, v. 35, n. 6, p. 1039-1042 - 2011. Disponível em: <http://dx.doi.org/10.1590/S1413-70542011000600001>. DOI: 10.1590/S141370542011000600001.

FREKING, B.A.; LENTS, C.A.; VALLET, J.L.. Selection for uterine capacity improves lifetime productivity of sows. Animal Reproduction Science, v. 167, p. 16-21 2016. Disponível em: <https://doi.org/10.1016/j.anireprosci.2016.01.018>. DOI: 10.1016 / j.anireprosci.2016.01.018.

GONZALEZ-ANOVER, P.; GONZALEZ-BULNES, A.. Maternal age modulates the effects of early-pregnancy L-proline supplementation on the birth-weight of piglets. Animal Reproduction Science, v. 181, p. 63-68 - 2017. Disponível em: < http://dx.doi.org/doi:10.1016/j.anireprosci.2017.03.016>. DOI: 10.1016 / j.anireprosci.2017.03.016.

GRUHOT, T.R.; CALDERÓN DÍAZ, J.A.; BAAS, T.J.; DHUYVETTER, K.C.; SCHULZ, L.L.; STALDER, K.K.. An economic analysis of sow retention in a United States breed-to-wean system. Journal Swine Health Production, v. 25, n. 5, p. 238-246 - 2017. $\quad$ Disponível em: https://www.aasv.org/shap/issues/v25n5/v25n5p238.html>.

GUIMARÃES, D.D.; AMARAL, G.F.; MAIA, G.B.S.; LEMOS, M.L.F.; ITO, M.; CUSTODIO, S.. Suinocultura: estrutura da cadeia produtiva, panorama do setor no Brasil e no mundo e o apoio do BNDES. BNDES Setorial, Rio de Janeiro, v. 85, n. 45, p.132-136 - $2017 . \quad$ Disponível em: <http://web.bndes.gov.br/bib/jspui/handle/1408/11794>.

KANEKO, M.; KOKETSU, Y.. Gilt development and mating in commercial swine herds with varying reproductive performance. Theriogenology, v. 77, p. 840-846 2012. Disponível em: <https://doi.org/10.1016/j.theriogenology.2011.09.006>. DOI: 10.1016 / j.theriogenology.2011.09.006.

KAPELAŃSKI, W.; JANKOWIAK, H.; BOCIAN, M.; GRAJEWSKA, S.; DYBAŁA, J.; MUDZIŃSKA, A.. Morphometric characteristics of the reproductive system in Polish Large White and Polish Landrace gilts at $100 \mathrm{~kg}$ body weight. Animal Science, v. 13, n. 1, p. 45-53 - 2013. Disponível em: <https://doi.org/10.2478/v10220-012-00578>. DOI: 10.2478/v10220-012-0057-8.

KNOX, R.V.; SHEN, J.; GREINER, L.L.; CONNOR, J.F.. Effect of timing of relocation of replacement gilts from group pens to individual stalls before breeding on fertility and well-being. Journal Animal Science, v. 94, p. 5114-5121 - 2016. Disponível em: <https://doi.org/10.2527/jas.2016-0799>. DOI: 10.2527/jas.2016-0799. 
KOKETSU, Y.; TANI, S.; IIDA, R.. Factors for improving reproductive performance of sows and herd productivity in commercial breeding herds. Porcine Health Management, v. 1, n. 3 - 2017. Disponível em: <https://doi.org/10.1186/s40813-0160049-7>. DOI: 10.1186/s40813-016-0049-7.

KUMMER, R.; BORTOLOZZO, F.P.; WENTZ, I.; BERNARDI, M.L.. Existe diferença no desempenho reprodutivo ao primeiro parto de leitoas inseminadas no $1^{\circ}, 2^{\circ}, 3^{\circ}$ ou $4^{\circ}$ estro?. Acta Scientiae Veterinariae, v. 33, p. 125-130 - 2005. Disponível em: < https://doi.org/10.22456/1679-9216.14783>. DOI: 10.22456/1679-9216.14783.

MORRISON, M.D.A. Estimates of variance componentes for gilt retention traits. MS Thesis, North Carolina State Univ., Raleigh - 2016. Disponível em: < http://www.lib.ncsu.edu/resolver/1840.16/11333>.

MOURA, C. A., 2016. "Pontos chave no povoamento de granjas produtoras de leitões". Suinocast (2016). Web 6 Mar/ 2018.

PINILLA, J.C.; LECZNIESKI, L.. Parity distribution management and culling. Manitoba Swine Seminar, v. 24 - 2010.

ROONGSITTHICHAI, A.; CHEUCHUCHART, P.; CHATWIJITKUL, $S$; CHANTAROTHAI, O.; TUMMARUK, P.. Influence of age at first estrus, body weight, and average daily gain of replacement gilts on their subsequent reproductive performance as sows. Livestock Science, v. 151, p. 238-245 - 2013. Disponível em: <https://doi.org/10.1016/j.livsci.2012.11.004>. DOI: 10.1016/j.livsci.2012.11.004.

ROONGSITTHICHAI, A.; OLANRATMANEE, E.; TUMMARUK, P.. Influence of growth rate and onset of boar contact on puberty attainment of replacement gilts raised in Thailand. Tropical Animal Health and Production, v. 46, p. 1243-1248 2014. Disponível em: <https://link.springer.com/article/10.1007\%2Fs11250-0140634-1>. DOI: 10.1007 / s11250-014-0634-1.

ROY, C.; LAVOIE, M.; RICHARD, G.; ARCHAMBAULT, A.; LAPOINTE, J.. Evidence that oxidative stress is higher in replacement gilts than in multiparous sows. Journal of Animal Physiology and Animal Nutrition, v. 100, p. 911-919 - 2016. Disponível em: <https://doi.org/10.1111/jpn.12462>. DOI: 10.1111/jpn.12462.

SAITO, H.; SASAKI, Y.; KOKETSU, Y.. Associations between Age of Gilts at First Mating and Lifetime Performance or Culling Risk in Commercial Herds. Journal Veterinary Medicine Science, v. 5, n. 73, p. 555-559 - 2011. Disponível em: <https://doi.org/10.1292/jvms.10-0040>. DOI: 10.1292/jvms.10-0040.

SEE, G.M.; GRUHOT, T.R.; SPANGLER, M.L.; LEWIS, R.M.. Longitudinal analysis of weight showed little relationship with age at puberty in gilts. American Society of Animal, v. 96, p. 4959-4966 - 2018. Disponível em: <https://doi.org/10.1093/jas/sky366>. DOI: 10.1093/jas/sky366.

SZULC, K.; SKRZYPCZAK, E.; BUCZYŃSKI, J.T.; GRACZYK, T.. Influence of body weight and age at first insemination of PIC gilts on the results of reproductive performance and piglet rearing. Scientific Annals of Polish Society of Animal 
Production, v. 11, n. 1, p. 49-57 - 2015. Disponível em: <http://rn.ptz.icm.edu.pl/wpcontent/uploads/2016/12/Szulc-ang.pdf>.

VIDOVIĆ, V.; ŠTRBAC, L.J.; LUKAČ, D.; STUPAR, M.. Influence of age and weight of landrace gilts at fertile insemination on litter size and longevity. Biotechnology in Animal Husbandry, v. 1, n. 27, p. 75-84 - 2011. Disponível em: <https://doi.org/10.2298/BAH1101075V>. DOI: 10.2298/BAH1101075V. 